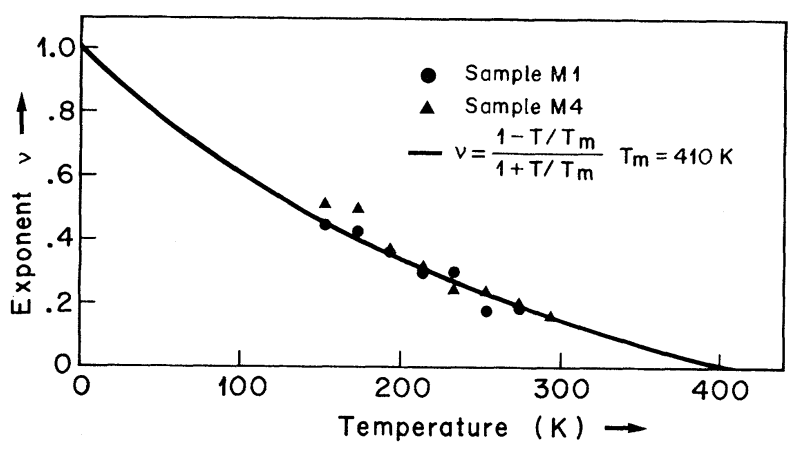

FIG. 2. Temperature dependence of the conductivity exponent $\nu$ [see Eqs. (16) and (17)].

the effective attempt frequency $f_{0^{\circ}}$ From a detailed analysis of the experimental data we deduce the values $L / a \lesssim 10, \Delta_{0} \sim 0.2 \mathrm{eV}$, and $f_{0} \sim 10^{11}$ $\mathrm{Hz}$. In addition, Eq. (19) and the experimental upper bound for $\sigma(0)$ of $10^{-9}(\Omega \mathrm{cm})^{-1}$ lead to a lower bound for $\Delta_{1}$ of the order of $2 \mathrm{eV}$.

In conclusion, we have demonstrated that a simple variable-barrier model can lead to interesting and unconventional predictions for the impurity-dominated transport properties of onedimensional systems. It is able to explain the anomalous power-law behavior of $\sigma(\omega)$ as well as the temperature dependence of the exponent $\nu$ observed in hollandite. A detailed fit to the experimental data, moreover, leads to reasonable and consistent values for the model parameters. To our knowledge no other model for classical transport in one-dimensional systems is able to predict this rather complex conductivity behavior.

The authors gratefully acknowledge helpful conversations with Professor R. Orbach。One of us (S.A.) would like to thank the Brown Boveri Research Center and the IBM Research Laboratory for their hospitality during the performance of this work.

\footnotetext{
${ }^{1}$ See, e.g., Lecture Notes in Physics 65, Organic Conductors and Semiconductors (Springer, New York, 1977); A. A. Abrikosov and I. A. Ryzhkin, Adv. Phys. 27, 147 (1978); M. J. Rice and J. Bernasconi, J. Phys. $\overline{\mathrm{F}} 2,905$ (1972), and $\underline{3}, 55$ (1973).

${ }^{2}$ See, e.g., H. Scher and E。W. Montroll, Phys. Rev. B 12, 2455 (1975); S。 R. Elliott, Philos。Mag. 36, 1291 (1977).

${ }^{3}$ A. Byström and A. M。 Byström, Acta Crystallogr. 3, $146(1950)$.

${ }^{4}$ H. U. Beyeler, Phys. Rev. Lett. 37, 1557 (1976).

${ }^{5}$ H. U. Beyeler and S. Strässler, unpublished.

${ }^{6} \mathrm{~J}$. Bernasconi, S. Alexander, and R. Orbach, Phys. Rev. Lett. 41,185 (1978)。

${ }^{7}$ Our $\Gamma_{\text {eff }}(\omega)$ formally corresponds to the Laplace transform of the relaxation function $\varphi(t)$ in the "continuous-time random-walk model" of Scher and Montroll (Ref. 2). We note, however, that the determination and the physical interpretation of $\Gamma_{\text {eff }}(\omega)$ is fundamentally different from their characterization of $\varphi(t)$ in terms of a site-independent hopping-time distribution.

${ }^{8} \mathrm{~S}$. Alexander and J. Bernasconi, to be published.
}

\title{
Charge Retention in Deep-Inelastic Electroproduction
}

R. Erickson, I. Cohen, F. Messing, ${ }^{(a)}$ E. Nordberg, R. Siemann, J. Smith-Kintner, and P. Stein Cornell University, Ithaca, New York 14853

and

G. Drews, W. Gebert, F. Janata, P. Joos, A. Ladage, H. Nagel, ${ }^{(b)}$ H. Preissner, and P. Söding II. Institut für Experimentalphysik der Universität Hamburg, and Deutsches Elektronen-Synchrotron (DESY), Hamburg, Germany

(Received 10 January 1979)

We have measured the net charge of the forward hadrons electroproduced from a proton target and have observed a rise with increasing $x\left(=Q^{2} / 2 M \nu\right)$. This effect is expected in the quark-proton model as the electroproduction of hadrons becomes dominated by the fragmentation of $u$ quarks. The data are also consistent with jet models in which a highmomentum leading hadron, rather than a slower hadron, is more likely to be carrying the parent quark.

The identification of final-state hadrons as the fragmentation products of fractionally charged quarks is an important but experimentally elu- sive feature of the quark-parton model. As a direct test of this feature, Feynman conjectured that the hadrons arising from quark fragmenta- 
tion would retain the charge of the parent quark when averaged over many events. ${ }^{1}$ This test would be straightforward if the quark and target fragmentation regions were separated by a plateau in the hadron rapidity distribution, but this would require an energy much higher than that currently available. At present energies, the quark and target fragmentation regions overlap by an amount that depends on the hadronic centerof-mass energy $W$. On the other hand, the relative proportions of different flavors, and hence the average charge, of the scattered quarks is a function of $x=Q^{2} / 2 M \nu$ (where $-Q^{2}$ and $\nu$ are the square of the mass and the energy of the virtual photon, respectively). Thus, if the overlap is fixed by holding $W$ constant, any $x$ dependence observed in the charge of the forward-going hadrons would be indicative of the charge-retention effect. In this Letter we report an observation of such $x$ dependence in a deep-inelastic electroproduction experiment. Our data can also be used to check the idea that a parent-quark flavor can be identified using the charge of the leading hadron. ${ }^{2}$

The experiment was performed at the Wilson Synchrotron Laboratory of Cornell University with an 11.5-GeV electron beam incident on a liquid-hydrogen target. The charged final-state hadrons were observed in a streamer chamber triggered by a scattered electron as discussed in Cohen et al.$^{3}$ The analysis described here is based on events in the region $1<Q^{2}<6 \mathrm{GeV}^{2}\left(\left\langle Q^{2}\right\rangle\right.$ $\left.=2.4 \mathrm{GeV}^{2}\right)$ and $2.5<W<4.2 \mathrm{GeV}(\langle W\rangle=3.2 \mathrm{GeV})$. Since the streamer chamber had nearly $4 \pi$ acceptance, all charged final-state particles were detected throughout a large kinematic region, minimizing systematic uncertainties。

In the quark-parton model, the mean net charge $\langle c\rangle$ in the quark fragmentation region is given by the sum of the charges of the parent quarks, each weighted by its contribution to the inelastic cross section but shifted by a universal constant $\eta$. This constant is the average charge of the antiquarks formed in the fragmentation process and must be added to the charge of the parent quark (or subtracted if the parent is an antiquark), because hadrons, and not individual quarks, are observed in the experiment ${ }_{0}^{4}$ Thus,

$$
\langle c\rangle=\frac{\sum e_{j}^{2}\left(e_{j} \pm \eta\right) q_{j}(x)}{\sum e_{j}^{2} q_{j}(x)},
$$

where $q_{j}(x)$ is the number density distribution of quark flavor $j$ with charge $e_{j}$ 。

Limiting values of $\eta$ would be zero if $u \bar{u}, d \bar{d}$, and $s \bar{s}$ pairs were equally probable, or $-\frac{1}{6}$ if strange quarks were completely suppressed. In an earlier publication, ${ }^{3}$ we reported a measurement of the relative probability of electroproducing strange or nonstrange hadrons. Using this result and assuming the ratio of vector to pseudoscalar meson production ${ }^{5}$ to be between 1 and 3 , we have calculated that $\eta$ falls between -0.12 and -0.09 .

We have measured $\langle c\rangle$ by including all hadrons (including protons) with $X_{F}=p_{\|} * / p_{\max } *$ greater than some suitably chosen value, where $p_{\|}{ }^{*}$ is the component of the hadron's momentum in the direction of the virtual photon and the asterisk indicates the hadronic center-of-mass frame. Since this experiment had no provision for distinguishing among hadrons, mass assignments necessary for the Lorentz transformation were made on a statistical basis using parametrizations of hadron structure functions measured in inclusive experiments. ${ }^{6}$ The dominant source of uncertainty in this procedure is the extrapolation of the available proton data from the energy region $2.0<W<3.1 \mathrm{GeV}$ to our energy range. ${ }^{7}$ As a check of these parametrizations, we compared the laboratory momentum distributions expected for the sum of positive pions, kaons, and protons to our measured positive track distributions and found excellent agreement in both the shape and normalization。 The mass-assignment procedure introduces $x$-independent uncertainties in $\langle c\rangle$ of $\pm 4 \%$ due to the kaons and $\pm 6 \%$ due to the protons. The loss of forward hadrons due to experimental inefficiences has a net effect on $\langle c\rangle$ of less than $5 \%$ 。

To test for other possible systematic problems, we repeated the analysis in more restricted $Q^{2}$ and $W$ ranges and also with more stringent electron identification criteria. Our standard criteria lead to a small contamination of $\pi^{-}$triggers ${ }^{3}$; the more stringent criteria reduced this problem to a negligible level. Results consistent with those presented below were obtained in each case, but with reduced statistical significance.

The charge $\langle c\rangle$ for $X_{F}>0$ is plotted in Fig. 1 as a function of $x$, along with points from photoproduction and muon-production experiments. The photoproduction point was obtained from fits to the $\pi^{+} / \pi^{-}$ratio, ${ }^{8 \cdot 9}$ the $\pi^{-}$production cross section, ${ }^{10}$ and the proton production cross section ${ }^{8}$ measured at energies close to ours. There is a systematic uncertainty in this point due to the lack of $\pi^{+} / \pi^{-}$and proton data for $p_{\perp}<0.2 \mathrm{GeV} / c$. The muon-production points were calculated from 


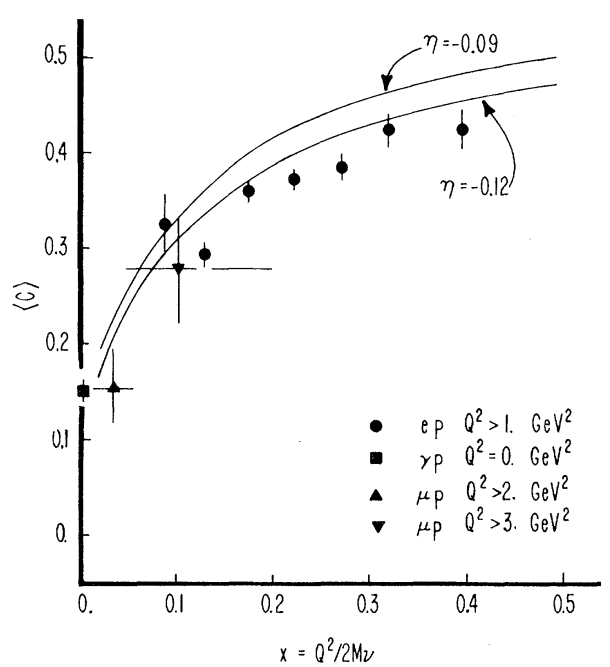

FIG. 1. The $x$ dependence of the mean charge of hadrons with $X_{F}>0$ is compared to a quark-parton model calculation. The photoproduction point is based on fits to the data of Refs. 8,9 , and 10, and the muon-production points are based on data from Ref. 11. The error bars show statistical uncertainties only. The curves are given by Eq. (1).

the charged-hadron data of Loomis et al.,$^{11}$ which we fitted with exponentials down to $X_{F}=0.08$ and extrapolated to $X_{F}=0$. The low- $x$ point is based on data at $Q^{2}>2 \mathrm{GeV}^{2}$ and $W>6.2 \mathrm{GeV}(\langle W\rangle \approx 12$ $\mathrm{GeV})$; the high- $x$ point on data at $Q^{2}>3 \mathrm{GeV}^{2}$ and $W>3.6 \mathrm{GeV}(\langle W\rangle \approx 9 \mathrm{GeV})$. These points were collected at a higher $W$ than the others, resulting in a smaller overlap of the quark and target fragmentation regions. Nevertheless, these measurements of $\langle c\rangle$ agree with ours in the $x$ range they share and follow the trend of the other experiments, indicating that even a moderate change in $W$ does not significantly alter our results.

The curves in Fig. 1 show the $x$ dependence of Eq. (1) using the quark distribution functions of Field and Feynman. ${ }^{12}\langle c\rangle$ is expected to rise with increasing $x$ as the interactions become dominated by valence $u$ quarks. The data are in agreement with this prediction.

The $x$-dependent trend of the data remains unchanged as the $X_{F}$ cut is raised (Fig. 2), confirming that the observed effect is not simply a consequence of the experimental definition of the quark fragmentation region. Raising this cut reduces the probability that the parent quark is included in the forward region, but does not affect the predicted $x$ dependence. The overall value of $\langle c\rangle$ falls because the average then includes events with no charged or neutral hadrons for-

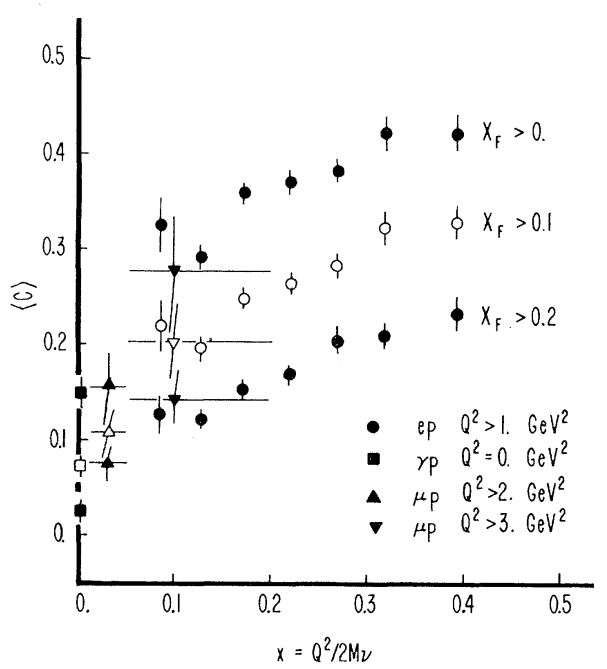

FIG. 2. The insensitivity of the $x$ dependence of the mean forward charge to the particular $X_{F}$ cut is checked for various cuts.

ward of the cut. A higher $X_{F}$ cut reduces the systematic undertainties in the photoproduction and muon-production points because extrapolations outside the $X_{F}$ range of the available data are not required.

To further examine the effect of various $X_{F}$ cuts, we have plotted the average total charge $\Gamma\left(X_{F}\right)$ forward of these cuts in Fig. 3 for events with $x>0.2$. We have included at each point only those events having at least one charged hadron

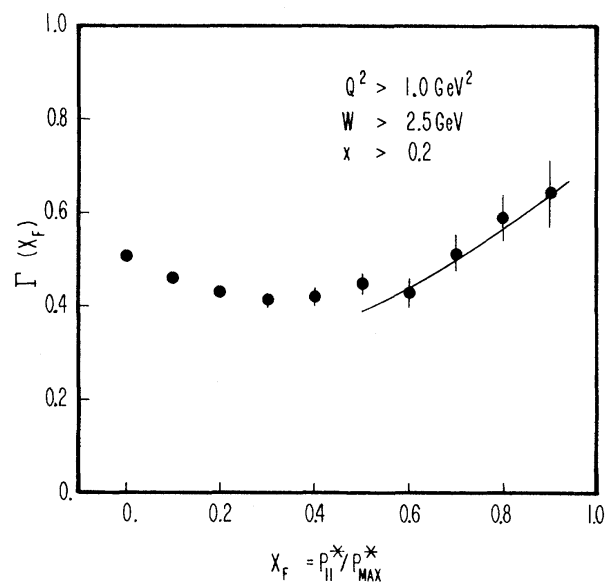

FIG. 3. The net charge forward of various $X_{F}$ cuts is plotted for events with $x>0.2$. Each datum point contains only events having at least one charged hadron forward of the corresponding $X_{F}$ value. The curve is the result of a Monte Carlo jet-model calculation. 
forward of the corresponding cut. $\Gamma\left(X_{F}\right)$ is seen to be independent of $X_{F}$ for $0<X_{F}<0.6$, showing that the overall drop seen when the cut is raised in Fig. 2 is due to an increase in the fraction of events with no forward charged hadrons, and not to a relative increase in the number of negative hadrons.

Figure 3 can also be used to test whether the charge of the leading particle is a clue to the identity of the parent quark, an idea that arises naturally from jet models ${ }_{\circ}{ }^{2}$ This can be checked for $X_{F}$ cuts greater than 0.5 , because there can be at most one hadron with $X_{F}>0.5$ in any event. For example, the value $0.45 \pm 0.03$ measured at $X_{F}>0.5$ indicates that $72 \%$ of the charged hadrons past this cut are positive. For $x>0.2(\langle x\rangle=0.28)$, approximately $87 \%$ of the total cross section is due to $u$ quarks and $11 \%$ is due to $d$ quarks. Neglecting sea-quark contributions, we then conclude that for jets initiated by $u$ quarks $80 \%$ of the charged hadrons with $X_{F}>0.5$ will be positive。

As the $X_{F}$ cut is increased further, the average charge of the leading particle rises. The curve in Fig. 3 is the result of a Monte Carlo jet simulation similar to the recursive scheme suggested by Field and Feynman, ${ }^{2}$ but modified to match our strange-particle distributions ${ }^{3}$ and energy range. For $X_{F}$ cuts less than 0.5 , the Monte Carlo simulation is sensitive to uncertainties in the latter modification, and nonleading hadrons contribute to our measurement of $\Gamma\left(X_{F}\right)$.

In conclusion, our data indicate that electroproduced hadrons in the quark fragmentation region retain the charge of the parent quarks. The agreement between our data and a simple quarkparton model is striking. We also find that the mean charge of high-momentum leading hadrons is consistent with the prediction of a quark-jet model.

This work was supported in part by the Bundesministerium für Forschung und Technologie and the National Science Foundation. We gratefully acknowledge the support of Professor B。 D. Mc-
Daniel, Professor H. Schopper, and Professor G. Weber, and the staffs of the Wilson Synchrotron Laboratory and Deutsches Electronen-Synchrontron。 We are indebted to J. T. Seeman for his assistance in constructing the detector and for his helpful comments during the analysis.

\footnotetext{
(a) Present address: Carnegie-Mellon University, Pittsburgh, Penn.

(b) Present address: Beiersdorf AG, Hamburg, Germany.

${ }^{1}$ R. P. Feynman, Photon-Hadron Interactions (Benjamin, Reading, Mass., 1972).

${ }^{2}$ R. D. Field and R. P. Feynman, Nucl. Phys. B136, 1 (1978).

${ }^{3}$ I. Coehn et al., Phys. Rev. Lett. 40, 1614 (1978).

${ }^{4}$ G. Farrar and J. Rosner, Phys. Rev. D 7, 2747 (1973); R. Cahn and E. Colglazier, Phys. Rev. D $\underline{9}$, 2658 (1974); J. L. Newmeyer and D. Sivers, Phys. Rev. D 9 , 2592 (1974); S. J. Brodsky and Nathan Weiss,
} SLAC Report No. SLAC-PUB-1926, 1977 (unpublished).

${ }^{5} \mathrm{G}$. Drews et al., in Proceedings of International Symposium on High Energy Physics, Tokyo, 1978 (unpublished).

${ }^{6}$ H. Ackermann et al., Nucl. Phys. B120, 365 (1977); L. Ahrens et al ., Phys. Rev。D 9, 1894 (1974); J. C. Alder et al., Nucl. Phys. B46, 415 (1972); C. J. Bebek et al., Phys. Rev. Lett. 32, 27 (1974); C. J. Bebek et al., Phys. Rev. Lett. 34, 1115 (1975); C. J. Bebek et al., Phys. Rev. D 15, 3077 (1977); C. J. Bebek et al., Phys. Rev. D 16, 1986 (1977); A. Browman et al., Phys. Rev. Lett. 37, 974 (1976); K. Hanson, in Proceedings of the Intermational Symposium on Lepton and Photon Interactions at High Energies, Stanford, California, 1975, edited by W. Kirk (Stanford Linear Accelerator Center, Stanford, Calif., 1976); J. F. Martin et al., Phys. Rev. Lett. 40,283 (1978).

${ }^{7}$ G. Drews et al., Phys. Rev. Lett. 41, 1433 (1978).

${ }^{8} \mathrm{H}$. Burfeindt et al., Nucl. Phys. B74, 189 (1974).

${ }^{9}$ W. Kaune et al., Phys. Rev. D 11, 478 (1975).

${ }^{10}$ K. C. Moffeit et al., Phys. Rev. D 5, 1603 (1972).

${ }^{11}$ W. A. Loomis et al., "Hadron Production in MuonProton and Muon-Deuteron Collisions" (to be published).

${ }^{12}$ R. D. Field and R. P. Feynman, Phys. Rev. D $\underline{15}$, 2590 (1977). 